\title{
Erratum to: Solution of Kepler's Equation with Machine Precision
}

\author{
M. K. Abubekerov ${ }^{a, *}$ and N. Yu. Gostev ${ }^{a, * *}$ \\ ${ }^{a}$ Sternberg Astronomical Institute, Moscow State University, Moscow, 119991 Russia \\ *e-mail:marat@sai.msu.ru \\ **e-mail:ngostev@mail.ru
}

Received May 25, 2021; revised May 25, 2021; accepted May 25, 2021

DOI: $10.1134 /$ S1063772921330015

Page 1066, Footnotes 1 and 2 should read as follows:

http://lnfm1.sai.msu.su/ ngostev/Files/Kepler.zip

$<$ http://lnfm1.sai.msu.su/ngostev/Files/Kepler.zip $>$

http://lnfm1.sai.msu.su/ ngostev/algorithm.html

$<$ http://lnfm1.sai.msu.su/ngostev/algorithm.html $>$

The original article can be found online at

https://doi.org/10.1134/S106377292012001X 